\title{
ADSORCIÓN DE MERCURIO Y CADMIO EN CARBÓN SUBBITUMINOSO OXIDADO-XANTADO, SULFONADO $O$ ACTIVADO Y RESINA SINTÉTICA COMERCIAL
}

\section{MERCURY AND CADMIUM ADSORPTION IN SUBBITUMINOUS XANTHATED, SULFONATED OR ACTIVATED CARBON AND COMMERCIAL SYNTHETIC RESIN}

\author{
Roberto Fernández-Maestre ${ }^{1}$, Fredy Colpas-Castillo ${ }^{2}$
}

${ }^{1}$ Químico, Ph.D., Programa de Química. Universidad de Cartagena, Campus de San Pablo, Cartagena de Indias, Bolívar, Colombia, e-mail: rfernandezm@unicartagena.edu.co; autor para correspondencia; ${ }^{2}$ Químico, M.Sc., Programa de Química. Universidad de Cartagena, Campus de San Pablo, Cartagena de Indias, Bolívar, Colombia, e-mail: fcolpasc@unicartagena. edu.co

Rev. U.D.C.A Act. \& Div. Cient. 18(1): 241-250, Enero- Junio, 2015

\section{RESUMEN}

La combustión del carbón de bajo rango produce impactos ambientales por su bajo calor de combustión y alto contenido de heteroátomos, lo que ha promovido sus usos alternativos, como adsorbente. Un carbón subbituminoso (CS), se oxidó con aire a $150^{\circ} \mathrm{C}$, en un lecho fijo, durante cuatro horas y se xantó con disulfuro de carbono en solución básica, a $30^{\circ} \mathrm{C}$. Este carbón xantado (CX), se evaluó para la adsorción de $\mathrm{Hg}^{2+}$ y $\mathrm{Cd}^{2+}$ de soluciones acuosas de 0,100 , $1,000,12,48,124,8$ y $998,3 \mathrm{mg} / \mathrm{L}$; los metales, se determinaron por espectrometría de absorción atómica. CX se comparó con CS, CS sulfonado y CS activado (CAp), además de un carbón activado comercial (CAC) y una resina sintética comercial (RC), en términos de su capacidad de adsorción de metales. RC mostró la más alta capacidad de adsorción (factor de concentración 98\%), seguido por CX (factor de concentración 96\%). En general, el $\mathrm{Hg}^{2+}$ se adsorbió menos que el $\mathrm{Cd}^{2+}$ y las capacidades de adsorción siguieron el orden, de mayor a menor: RC, CX, CAp, CS, y CAC. CX también mostró una mejor adsorción de $\mathrm{Cd}^{2+}, 81 \%$ vs. $15 \%$, que un carbón xantado no oxidado, lo que muestra que la oxidación previa del carbón aumentó la cantidad de grupos oxigenados que, a su vez, aumentaron la xantación.

Palabras clave: Adsorción, carbón activado, carbón subbituminoso, xantación, espectrometría de absorción atómica.

\section{SUMMARY}

The combustion of low rank coal produces environmental impacts due to its low heat of combustion and high heteroatom content which has promoted its alternative uses as an adsorbent. A subbituminous coal (CS) was oxidized with air at $150^{\circ} \mathrm{C}$ on a fixed bed for 4 hours and xanthated with carbon disulfide in a basic solution, at $30^{\circ} \mathrm{C}$. The resulting xanthated coal (CX) was evaluated for the adsorption of $\mathrm{Hg}^{2+}$ and $\mathrm{Cd}^{2+}$ from $0,100,1,000,12.48$, 124.8 y $998.3 \mathrm{mg} / \mathrm{L}$ aqueous solutions; metal concentrations were determined by atomic absorption spectrometry. CX was compared against CS, a sulfonated CS, activated CS (CAp), a commercial activated carbon (CAC), and a commercial synthetic resin (RC) in terms of $\mathrm{Hg}^{2+}$ and $\mathrm{Cd}^{2+}$ adsorption. $\mathrm{RC}$ showed the highest adsorption capacity (concentration factor 98\%) followed by theCX (concentration factor 96\%). The adsorption of cadmium on the sulfonated CS was $0.56 \mathrm{meq} / \mathrm{g}$, lower than that of CX, $1.85 \mathrm{meq} / \mathrm{g}$. In general, $\mathrm{Hg}^{2+}$ was less adsorbed than $\mathrm{Cd}^{2+}$; the adsorption capacities decreased in the order: RC, CX, sulfonated CS, activated CS, CS, and CAC. CX showed a better $\mathrm{Cd}^{2+}$ adsorption, $81 \%$ against $15 \%$, than a non-oxidized 40 -hours-xanthated coal, which shows that oxidation of coal increased the amount of oxygenated groups that enhanced xanthation.

Key words: Adsorption, brown coal, activated carbon, subbituminous coal, xanthation, atomic absorption spectrometry.

\section{INTRODUCCIÓN}

De acuerdo a la World Coal Association (2014), la producción mundial de carbón, en el 2012, ascendió a más de 6.185 millones de toneladas, la de carbón de bajo rango, como lignito y carbón marrón, a 1.042 millones de toneladas y en Colombia, la de hulla, a unas 82 millones de toneladas. La mayor parte del carbón colombiano, se exporta para la generación de electricidad y para la producción de acero, de cemento y de combustible líquido. 
La combustión de carbón genera problemas ambientales, incluyendo la emisión de dióxido de carbono y cenizas volantes (Sun et al. 2009). La combustión de carbones de bajo rango origina más problemas medioambientales que la de carbones duros, debido a su bajo calor de combustión y alto contenido de heteroátomos, que introducen ácidos a la atmósfera, durante la combustión; los ácidos en el aire son responsables de la destrucción de monumentos y de edificios, daños a ríos, lagos, bosques, vegetación, ecosistemas terrestres y a la salud humana y disminución de la visibilidad (Jain et al. 2012). Estos problemas han promovido la investigación alternativa del carbón de bajo rango, como en intercambiadores de iones baratos que se pueden utilizar para desmineralización y descontaminación de aguas y recuperación de metales valiosos de soluciones de desecho (Castellar et al. 2013).

Los carbones de rango bajo, como lignito y subbituminosos, presentan propiedades naturales de adsorción, debido a grupos carboxílicos y fenólicos unidos a una estructura aromática, altamente reticulada (Hayashi \& Li, 2004). El uso de carbones de bajo rango para la adsorción de contaminantes orgánicos e inorgánicos, incluyendo metales pesados, continúa atrayendo el interés de la comunidad científica. Li \& Helmreich (2014) usaron carbón activado y lignito activado para adsorber compuestos orgánicos y zinc y Lao-Luque et al. (2014) investigaron la adsorción de Cr(III) presente en soluciones acuosas, usando leonardita, un producto de bajo costo de oxidación del lignito. Por otro lado, Rao et al. (2011) utilizaron lignito para adsorber $\mathrm{Pb}^{2+}$ de soluciones acuosas y se logró un $98 \%$ de adsorción.

Marañón \& Sastre (1992) aumentaron la capacidad de adsorción de pulpa de manzana, mediante el incremento del contenido de grupos funcionales, a través de reacciones de xantación; la xantación de la pulpa de manzana, un residuo procedente de la producción de sidra formada por biopolímeros, como celulosa y lignina, amplió su estabilidad estructural y el rendimiento, como intercambiador de iones. La formación de xantatos puede ser esquematizada como (Bailey et al. 1999):

$$
\mathrm{CS}_{2}+\mathrm{R}-\mathrm{OH} \stackrel{\mathrm{OH}^{-}}{\longrightarrow} \mathrm{RCS}_{2}{ }^{-}+\mathrm{H}_{2} \mathrm{O}
$$

La xantación aumenta el contenido de grupos funcionales complejantes de metales, a través de electrones libres enlazantes en el grupo CS2 (Haenel, 1992) y los xantatos resultantes adsorben metales, por medio de procesos de complejación, intercambio iónico o una mezcla de ambos (Kim \& Lee, 1998; Liang et al. 2009).

En la oxidación del carbón existen reacciones químicas entre el agente de oxidación y los grupos funcionales del carbón que incrementan el contenido de grupos carboxilo y oxígeno (Shi et al. 2012), requerido para la reacción con grupos xantatos. En este trabajo, se estudió la influencia de la oxidación con aire, temperatura de xantación y tamaño de partícula en las propiedades de adsorción de cadmio y de mercurio de un adsorbente, CX, obtenido por xantación de carbones subbituminosos de Montelíbano, Córdoba, Colombia; la capacidad de adsorción de CX se comparó con las del carbón subbituminoso original (CS), CS sulfonado y CS activado, además de carbón activado comercial (CAC) y resina comercial Maratón Dowex, RC, (Dow Chemical). Estos estudios son esenciales en Colombia, para buscar la utilización del carbón de bajo rango en actividades diferentes a aquellas altamente contaminantes, como la producción de energía y de acero.

\section{MATERIALES Y MÉTODOS}

Precauciones. El disulfuro de carbono es un compuesto tóxico que debe ser manejado en campanas de extracción, usando gafas, careta, bata, mandil y guantes. Este compuesto es muy inflamable e irritante al contacto con la piel, ojos y mucosas (ATDSR, 2014).

En el presente trabajo, se obtuvieron dos adsorbentes, CS sulfonado y CX, a partir de carbón subbituminoso de Montelíbano, Córdoba, Colombia y sus propiedades de adsorción, se compararon con las de otros adsorbentes (CS, CAp, CAC, y RC). RC (Maratón Dowex, de Dow Chemical) posee grupos de ácido sulfónico quelante (Lenntech, 2014); CAp, se obtuvo a partir de bagazo de caña de azúcar y tenía un área superficial de $369 \mathrm{~m}^{2} / \mathrm{g}$ y acidez de $1,68 \mathrm{meq} / \mathrm{g}$ (Colpas-Castillo et al. 2011); CAC era un material granular grado especial lavado con ácido producido por activación de carbón de lignito con vapor de agua marca Darco MRX, con tamaño de partícula de 0,60 a $2,00 \mathrm{~mm}$, área BET $613 \mathrm{~m}^{2} / \mathrm{g}$ y volumen total de poros $0,62 \mathrm{~cm}^{3} / \mathrm{g}$. El calor de combustión de CS, 9,925BTU/Lb y los análisis próximo y último de CS y CAp, se obtuvieron en la empresa SGS (Barranquilla, Colombia), de acuerdo con las normas ASTM, para el análisis de carbón (Tabla 1).

Las muestras de CS, se almacenaron en bolsas de polietileno después de su colecta, se molieron en morteros de porcelana y se tamizaron a tamaños de partícula, entre 0,1 y $2,5 \mathrm{~mm}$. Luego, se desmineralizaron con $\mathrm{HCl} 0,5 \mathrm{~N}$ durante $6 \mathrm{~h}$, se enjuagaron con agua destilada, hasta que alcanzó $\mathrm{pH} 6$ y se secaron a $105^{\circ} \mathrm{C}$, durante $2 \mathrm{~h}$ (Anillo-Correa et al. 2013). Se obtuvieron tres grupos de partículas de carbón, T1, T2 y T3, con tamaños: 0,1 $\geq \mathrm{T} 1<0,5 ; 0,5 \geq \mathrm{T} 2<1,0$; y $1,0 \geq \mathrm{T} 3<2,5 \mathrm{~mm}$. Las muestras, se extrajeron y se hincharon con tetrahidrofurano durante 3 días, se lavaron con agua destilada y se secaron a $105^{\circ} \mathrm{C}$, durante $2 \mathrm{~h}$; en presencia de disolventes de hinchamiento, las moléculas de carbón se disocian, reorganizan y vuelven a asociarse en conformaciones 
Tabla 1. Análisis elemental y próximo de CAp y CS.

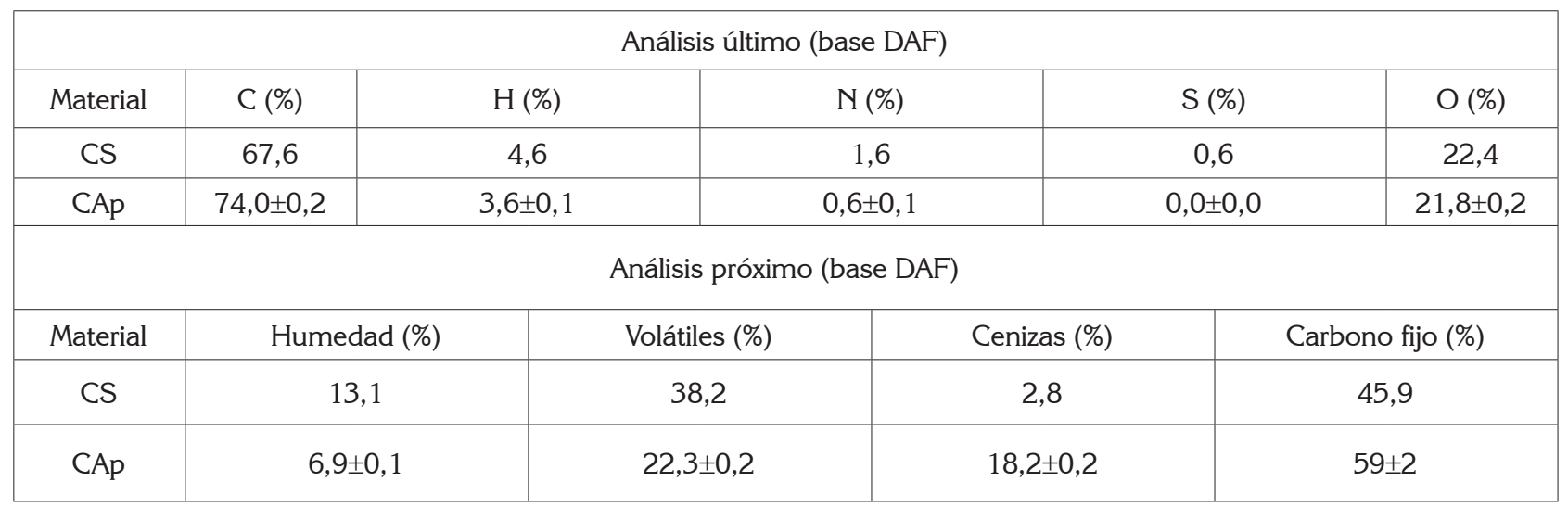

CS: Carbón subbituminoso original; CAp: Carbón activado preparado a partir de CS.

de menor energía, se rompen enlaces y se crean macroporos, lo que aumenta la difusión y forma sitios activos (Pinto et al. 1999). Este efecto incrementa el volumen del carbón que puede reaccionar para formar xantatos

Los experimentos, se realizaron en dos etapas. En la primera, se determinaron las condiciones óptimas para la xantación (temperatura y tamaño de partícula), xantando por 40h; se preparó CS sulfonado y se compararon las capacidades de adsorción de los carbones xantados y CS sulfonado; en la segunda etapa, se preparó CX: el carbón se oxidó con aire y se xantó usando las mejores condiciones encontradas en la primera etapa (tamaño de partícula $\mathrm{T} 1$ y $30^{\circ} \mathrm{C}$ ) y su capacidad de adsorción, se comparó con la de los otros adsorbentes.

Primera etapa. Los experimentos se llevaron a cabo por duplicado para cada tamaño de partícula de carbón y de temperatura de reacción. Para la xantación, se añadió al carbón una solución $6 \mathrm{M}$ de $\mathrm{NaOH}$ y después de $5 \mathrm{~min}$, $\mathrm{CS}_{2}$, en una proporción 1:1 molar, con respecto al $\mathrm{NaOH}$ y 3:1, respecto a la cantidad de $\mathrm{OH}$ en el carbón calculado, considerando que el oxígeno del carbón era igual a su contenido de $\mathrm{OH}$ (Wing et al. 1975). Después, la mezcla se agitó magnéticamente, durante $40 \mathrm{~h}$, a 30 o $7^{\circ} \mathrm{C}$.

Para la sulfonación, se utilizó ácido sulfúrico al $98 \%$, a $150^{\circ} \mathrm{C}$, durante 75 minutos, en una proporción 1:4, entre el ácido y el carbón tamaño T1, que fue el que arrojó los mejores resultados entre los carbones xantados. Por último, el carbón se lavó con agua destilada, hasta un $\mathrm{pH}$ mayor de 6 y se secó a $105^{\circ} \mathrm{C}$, durante 24 horas.

En los experimentos de adsorción con carbones xantados y CS sulfonado, $50,00 \mathrm{~mL}$ de soluciones de $7000 \mathrm{mg} / \mathrm{L}$ de $\mathrm{Cd}^{2+}$, se mezclaron con $0,5000 \mathrm{~g}$ de carbón xantado y CS sulfonado por triplicado, para cada combinación de tamaño de partícula y de temperatura de reacción. Después de agitar durante 24h (Torres-Blancas et al. 2013), el filtrado y eluido, se analizaron por espectrometría de absorción atómica, con llama para $\mathrm{Cd}^{2+}$ y por vapor frío, para $\mathrm{Hg}^{2+}$.

Segunda etapa. El carbón T1, se oxidó con aire a $150^{\circ} \mathrm{C}$, en un lecho fijo, durante $4 \mathrm{~h}$; se xantó siguiendo el procedimiento utilizado en la primera etapa, pero a temperatura ambiente durante 8h. Posteriormente, el carbón se lavó a pH 7 y se secó a $50^{\circ} \mathrm{C}$, durante $20 \mathrm{~h}$ (Anillo-Correa et al. 2013).

Para los experimentos de adsorción, 50,00mL de soluciones

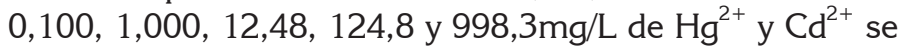
agitaron con $0,5000 \mathrm{~g}$ de CX, CS, CAp, RC y CAC, a pH 8, utilizando una solución tampón de carbonato-bicarbonato. Después de $6 \mathrm{~h}$ de agitación, los adsorbentes se filtraron y se eluyeron con $\mathrm{HCl} 0,5 \mathrm{~N}$ hasta completar $50,00 \mathrm{ml}$ de eluato. Las soluciones eluidas, se analizaron por espectrometría de absorción atómica con llama, para $\mathrm{Cd}^{2+}$ y por vapor frío, para $\mathrm{Hg}^{2+}$.

La capacidad de adsorción, se expresó como factor de concentración, FC, de acuerdo con Fehrmann \& Pohl (1993), definido como la relación de la masa adsorbida del metal en mg por gramo de adsorbente a la concentración de la solución catiónica, usando la fórmula:

$$
F C=\frac{\left(V_{i} C_{i}\right)-\left(V_{f} C_{f}\right)}{M C_{i}}
$$

Donde, Vi es el volumen de la solución inicial en $\mathrm{mL}$, Vf el volumen del filtrado en $\mathrm{mL}, M$ la masa de la muestra en $\mathrm{g}$, Ci la concentración metálica en la solución inicial en $\mathrm{mg} / \mathrm{L}$, Cf la concentración metálica en el filtrado en $\mathrm{mg} / \mathrm{L}$. En las condiciones de este trabajo, el mayor valor de FC es 100, el 
porcentaje de metal adsorbido, por lo que lo expresaremos como porcentaje. Los FC, se usaron para mostrar el inicio de la saturación de los adsorbentes con el aumento de la concentración de las soluciones. La saturación se alcanza cuando todos los sitios de adsorción del adsorbente están ocupados y comienza, cuando los factores de concentración disminuyen significativamente a menos del $100 \%$; el uso de concentraciones elevadas permite observar cuándo comienza la saturación para determinado metal; la saturación se incrementa a concentraciones más elevadas, ya que hay un equilibrio entre la concentración de metal en la solución y el número de sitios disponibles para adsorción.

Instrumentos y reactivos. Para los experimentos de extracción e hinchamiento, se usó tetrahidrofurano grado analítico y para la xantación, $\mathrm{NaOH}$ y $\mathrm{CS}_{2}$ grado comercial. Las soluciones de cadmio y de mercurio se prepararon a partir de reactivos analíticos, $\mathrm{CdCl}_{2}$ y $\mathrm{Hg}\left(\mathrm{NO}_{3}\right)_{2}$, suministrados por Merck. Para los análisis de absorción atómica con llama y vapor fue utilizado un instrumento Perkin Elmer Analyst 300. Los espectros infrarrojos, se tomaron en un instrumento FTIR Nicolet 5DXC.

\section{RESULTADOS Y DISCUSIÓN}

Se preparó un carbón subbituminoso xantado para adsorber $\mathrm{Cd}^{2+}$ y Hg$^{2+}$ de soluciones acuosas, se estudió el efecto de la temperatura de xantación, tamaño de partícula y la oxidación con aire en su capacidad de adsorción y se comparó este adsorbente con los otros utilizados. El examen de los resultados de los análisis próximo y último (Tabla 1) muestra que el contenido de carbono se incrementó de $68 \%$-en CS-, a 74\% —en CAp-, lo que es de esperarse, debido al tratamiento térmico al que se ha sometido CAp; este trata- miento igualmente disminuyó el contenido de volátiles, de 38 a $22 \%$, y la humedad, de 13 a 6,9\% y aumentó el contenido de cenizas, de 2,8 a $18 \%$ y de carbono fijo, de $46 \%$ a $59 \%$, por las altas temperaturas alcanzadas en este proceso. El análisis elemental nos da un indicio del contenido de grupos funcionales y de las posibles aplicaciones que se le pueden dar a los carbones. El alto contenido de oxígeno de los carbones subbituminosos y el incremento que se espera en estos después de la oxidación los hace ideales para la introducción de grupos xantatos. El calor de combustión es un parámetro importante para la clasificación de carbones, de acuerdo a las normas ASTM; el calor de combustión de CS, 9925BTU/Lb, indica que es un carbón de medio rango en cuanto a su poder calorífico, por lo que se puede utilizar para combustión o para usos alternativos.

Los espectros de infrarrojos, en la figura 1, muestran una banda de $\mathrm{CS}_{2}$ cerca de $1400 \mathrm{~cm}^{-1}$; además, una banda de estiramiento de $\mathrm{OH}$ fenólico, un poco más débil, en $3184 \mathrm{~cm}^{-1}$, indica que más grupos $\mathrm{OH}$ reaccionaron a baja temperatura; este comportamiento, se puede atribuir a un mayor rendimiento de la reacción a baja temperatura, debido a que el disulfuro de carbono es muy volátil a $30^{\circ} \mathrm{C}$ y su volatilización disminuye la cantidad de reactivo requerido para completar la xantación. Se pueden observar otros picos, entre 2900 y $3000 \mathrm{~cm}^{-1}$, producidos por estiramientos $\mathrm{C}-\mathrm{H}$, alrededor de $1600 \mathrm{~cm}^{-1}$, debido a estiramientos del doble enlace de grupos carboxilos y entre 1000 y $1100 \mathrm{~cm}^{-1}$, a vibraciones del doble enlace $\mathrm{C}=\mathrm{S}$ (Torres-Blancas et al. 2013).

En la primera etapa del estudio, el carbón xantado a $7^{\circ} \mathrm{C}$ mostró una FC promedio para cadmio, de $13 \pm 2$, para todos los tamaños de partícula, que no fue significativamente mayor $(p<0,05)$ que la del carbón xantado a $30^{\circ} \mathrm{C}(12 \pm 1)$

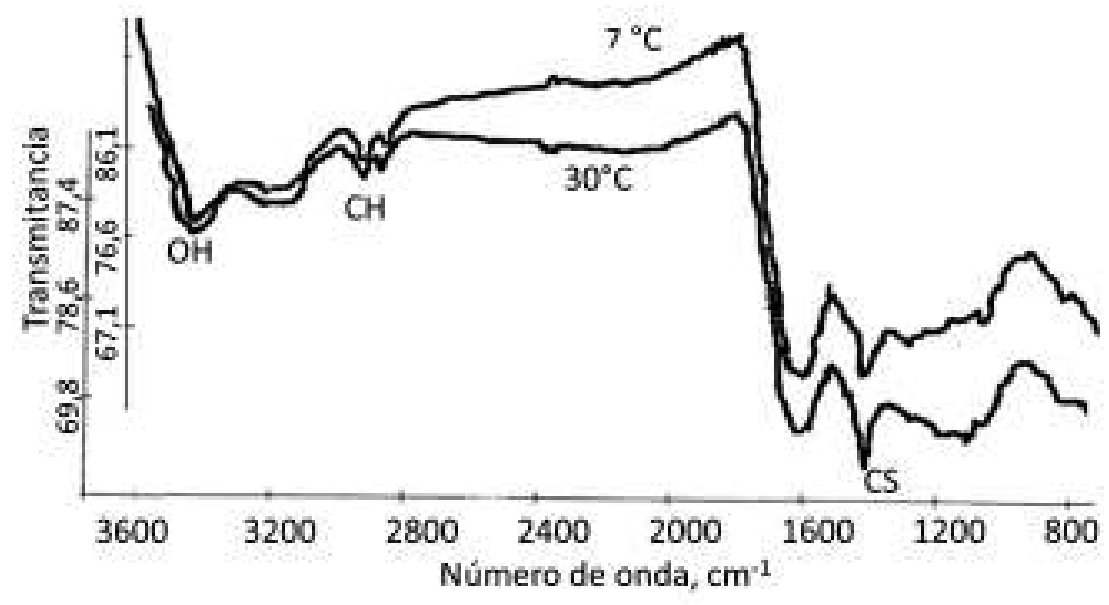

Figura 1. Espectro FT-IR de carbón xantado a 7 o $30^{\circ} \mathrm{C}$. El primer eje $y$ corresponde al espectro a $7^{\circ} \mathrm{C}$. 
(Tabla 2); además, el FC más alto para cadmio $(15,0)$ para el carbón xantado a $7^{\circ} \mathrm{C}$, se obtuvo para el tamaño $\mathrm{T} 1$, significativamente mayor $(\mathrm{p}<0,05)$, que el FC, de 12,0 del carbón de tamaño T2 o el FC de 11.6, para el T3. Con base en estos resultados, en la segunda etapa de los experimentos la xantación, se realizó con el carbón de tamaño $\mathrm{T} 1$, a $30^{\circ} \mathrm{C}$, ya que la mejor adsorción se obtuvo con el carbón de menor tamaño de partícula y hubo poca influencia de la temperatura de xantación, en la capacidad de adsorción; los mejores resultados de adsorción para los carbones con menor tamaño de partícula se explican, debido a que mientras más pequeño sea este, mayor será la capacidad de los carbones para adsorber cadmio, debido al aumento en el área superficial, obtenido con partículas más pequeñas (Sánchez-Soto, 2009), que exponen más grupos xantato para la adsorción de metales. Matsui et al. (2014) también encontraron que disminuyendo el tamaño de partícula de carbones activados se puede incrementar el equilibrio de adsorción de pequeñas moléculas y microcontaminantes.
El desempeño de CX, xantado con las mejores condiciones encontradas en la primera etapa de los experimentos, se comparó con las de CS, CS sulfonado, CAp, CAC, y RC. La adsorción de cadmio de una solución de 7000 ppm en CS sulfonado $(0,56 \mathrm{meq} / \mathrm{g})$, fue baja para un carbón T1 y mostró una diferencia significativa con la de CX $(1,85 \pm 0,09 \mathrm{meq} / \mathrm{g})$ $(p<0,05)$. Para los adsorbentes diferentes a CS sulfonado, las capacidades de adsorción para mercurio y cadmio de soluciones entre 0,1 y 998 ppm de $\mathrm{Hg}^{2+}$ y $\mathrm{Cd}^{2+}$ se muestran en la figura 2 y la tabla 3 , y se expresan como FC.

Las gráficas de concentración de la solución contra FC (Figura 2) muestran un comportamiento similar para todos los adsorbentes: los FC promedio de todos los adsorbentes disminuyeron con el aumento de las concentraciones de las soluciones; los FC promedio de ambos metales fueron $98,7 \pm 0,9 \%, 97 \pm 4 \%, 83 \pm 19 \%, 58 \pm 37 \%, 38 \pm 43 \%$ para $50,00 \mathrm{~mL}$ de soluciones $0,100,1,000,12,48,124,8$ y $998,4 \mathrm{mg} / \mathrm{L}$, respectivamente. Las pequeñas desviaciones de

Tabla 2. Capacidad de adsorción de carbón xantado por $40 \mathrm{~h}$ y temperatura de xantación de $7^{\circ} \mathrm{C}$, según tamaño de partícula. Los valores a $30^{\circ} \mathrm{C}$, se encuentran entre paréntesis.

\begin{tabular}{|c|c|c|c|}
\hline Intervalo de tamaño de partícula del carbón xantado por 40h & $0,1-0,5 \mathrm{~mm}$ & 0,5-1,0mm & $1,0-2,5 \mathrm{~mm}$ \\
\hline Factor de concentración & $15,0(13,5)$ & $12,0(11,2)$ & $11,6(10,9)$ \\
\hline Capacidad de adsorción, meq/g & $\begin{array}{r}1,85 \pm 0,09 \\
(1,68 \pm 0,08)\end{array}$ & $\begin{array}{r}1,46 \pm 0,05 \\
(1,37 \pm 0,04)\end{array}$ & $\begin{array}{r}1,43 \pm 0,06 \\
(1,32 \pm 0,05)\end{array}$ \\
\hline Capacidad de adsorción, mg/g & $\begin{array}{c}122 \pm 6 \\
(111 \pm 6)\end{array}$ & $\begin{array}{c}96 \pm 3 \\
(90 \pm 3)\end{array}$ & $\begin{array}{l}94 \pm 4 \\
(87 \pm 3)\end{array}$ \\
\hline
\end{tabular}

$50,00 \mathrm{~mL}$ de soluciones de $7000 \mathrm{mg} / \mathrm{L}$ de $\mathrm{Cd}^{2+}$ se mezclaron con $0,5000 \mathrm{~g}$ de carbón xantado por 40h por triplicado.
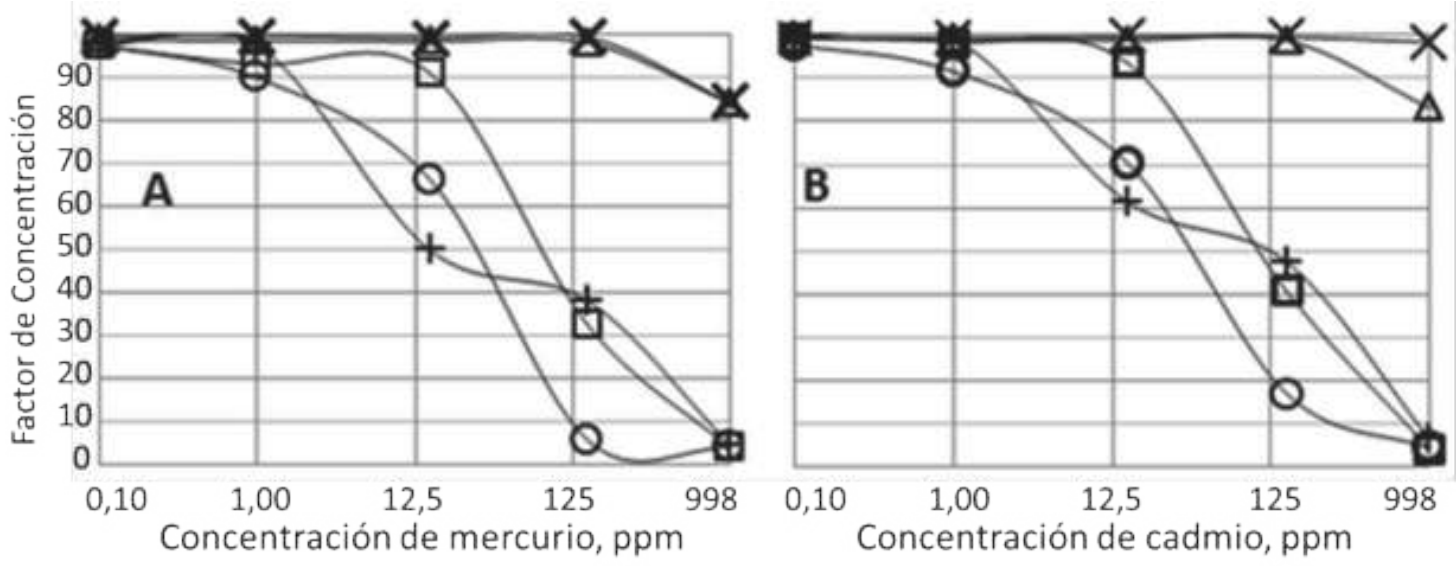

Figura 2. Capacidades de adsorción de mercurio (A) y cadmio (B) expresadas como FC. - - - CS; -o- CAC; -D-CX; -X- RC; -+CAp. 
Tabla 3. Comparación de FC de $\mathrm{Cd}^{2+} \mathrm{y} \mathrm{Hg}^{2+}$ para varios tipos de adsorbentes.

\begin{tabular}{|c|c|c|c|c|c|c|c|c|c|c|}
\hline \multirow{3}{*}{$\begin{array}{c}\text { Concentración } \\
\text { metálica, } \\
\text { mg/L }\end{array}$} & \multicolumn{10}{|c|}{ Adsorbentes } \\
\hline & \multicolumn{2}{|c|}{ CAC } & \multicolumn{2}{|c|}{ CS } & \multicolumn{2}{|c|}{ CAp } & \multicolumn{2}{|c|}{$\mathrm{RC}$} & \multicolumn{2}{|c|}{$C X$} \\
\hline & $\mathrm{Hg}$ & $\mathrm{Cd}$ & $\mathrm{Hg}$ & $\mathrm{Cd}$ & $\mathrm{Hg}$ & $\mathrm{Cd}$ & $\mathrm{Hg}$ & $\mathrm{Cd}$ & $\mathrm{Hg}$ & $\mathrm{Cd}$ \\
\hline 0,100 & 97,8 & 97,8 & 97,4 & 99,4 & 98,0 & 99,6 & 99,9 & 99,3 & 99,0 & 99,2 \\
\hline 1,000 & 90,0 & 91,6 & 93,2 & 98,4 & 97,6 & 99,3 & 99,9 & 99,3 & 98,6 & 99,0 \\
\hline 12,48 & 66,3 & 70,4 & 91,1 & 93,6 & 49,8 & 61,4 & 99,4 & 99,6 & 98,5 & 98,8 \\
\hline 124,8 & 5,6 & 17,0 & 32,5 & 40,7 & 38,1 & 47,5 & 99,5 & 99,5 & 98,2 & 98,8 \\
\hline 998,4 & 3,8 & 4,3 & 3,7 & 4,0 & 4,6 & 6,3 & 84,4 & 98,4 & 84,2 & 83,1 \\
\hline
\end{tabular}

Soluciones de 50,00mL de $\mathrm{Hg}^{2+}$ y Cd $^{2+}$ se agitaron con 0,5000g de los adsorbentes. CAC carbón activado comercial; CS carbón subbituminoso original; CAp carbón activado preparado a partir de carbón subbituminoso; RC: resina comercial; CX: carbón oxidado-xantado a $30^{\circ} \mathrm{C}$ con tamaño de partícula $\mathrm{T} 1$.

los dos primeros datos indican que los materiales adsorbieron prácticamente todo el metal de la solución y las elevadas desviaciones de los tres últimos reflejan las enormes diferencias en adsorción, debidas a la saturación de los materiales cuando las concentraciones aumentaron. Además, se observó el comienzo de la saturación con las soluciones de altas concentraciones de metal. La saturación de $0,5000 \mathrm{~g}$ de CAp, CS y CAC empezó con las soluciones de $998,4 \mathrm{mg} / \mathrm{L}$, para ambos metales. La tabla 4 compara las capacidades de adsorción de diferentes adsorbentes y, en ella, se observa que los carbones activados comerciales muestran capacidades de adsorción bajas, tal como las obtenidas en nuestro trabajo, para CAC. Se puede observar también en esta tabla, que los CA preparados a partir de carbón presentan mayores capacidades de adsorción que aquellos provenientes de materiales vegetales o cenizas; además, las capacidades de adsorción de nuestros CX fueron comparables e, incluso, superiores a muchas de las reportadas, obtenidos con materiales preparados a partir de carbón, indicando la calidad del producto fabricado.

En general, los FC mostrados por los adsorbentes fueron mayores para cadmio que para mercurio, aunque muy cercanos (Tabla 2), tal vez debido al pequeño tamaño iónico de $\mathrm{Cd}^{2+}$, que permitió al metal llegar a los poros más pequeños del carbón. CAC, CAp y CS mostraron menor capacidad para adsorber los metales pesados de soluciones acuosas, que RC y CX; esto se ejemplifica en las soluciones de concentración $998,4 \mathrm{mg} / \mathrm{L}$, para ambos metales, que en CAp, CAC y CS, que mostraron un FC promedio de $4 \pm 1 \%$, mientras que $\mathrm{FC}$ fue de $88 \pm 7 \%$ en promedio en $\mathrm{RC}$ y CX; RC mostró un FC promedio de $98 \pm 2 \%$ más alto que el del CX, $95,7 \pm 0,1 \%$, para las diez soluciones metálicas de diferentes concentraciones de ambos metales.
CX mostró una mayor adsorción de $\mathrm{Cd}^{2+}$ que un carbón xantado por más tiempo ( $40 \mathrm{~h}$ ) no oxidado ( $81 \%$ contra $15 \%)$, lo que sugiere que la oxidación del carbón aumenta la cantidad de grupos funcionales oxigenados en la estructura del carbón; esta oxigenación aumenta el grado de xantación y la densidad de los grupos adsorbentes en la superficie del carbón. Esto también implica que, para una mejor capacidad de adsorción del carbón, es más importante una oxidación previa para aumentar los grupos oxigenados que un largo tiempo de xantación.

En resumen, se oxidó y xantó un carbón subbituminoso de Montelíbano, Colombia, con disulfuro de carbono en solución básica, a $30^{\circ} \mathrm{C}$. La capacidad de adsorción del carbón xantado (CX) aumentó cuando se oxidó y el tamaño de partícula disminuyó hasta mostrar una capacidad de adsorción parecida a la de la resina comercial (RC). En general, el $\mathrm{Hg}^{2+}$ se adsorbió menos que el $\mathrm{Cd}^{2+}$. Las capacidades de adsorción para los adsorbentes siguieron este orden, de mayor a menor: RC, CX, CAp, CS, CAC. La xantación de carbones es un procedimiento promisorio para la preparación de materiales de desecho, orientado a la adsorción de contaminantes del ambiente y representa una alternativa a la costosa disposición de estos residuos en basureros.

Agradecimientos a SGS (Barranquilla, Colombia). Conflictos de intereses: El manuscrito fue preparado y revisado por todos los autores, quienes declaramos que no existe conflicto de intereses que ponga en riesgo la validez de los resultados presentados. 
Tabla 4. Comparación de la capacidad de adsorción para mercurio y cadmio de CX con reportes de la literatura.

\begin{tabular}{|c|c|c|c|}
\hline \multirow[t]{2}{*}{ Adsorbentes } & \multicolumn{2}{|c|}{$\begin{array}{l}\text { Capacidad de } \\
\text { adsorción, mg/g }\end{array}$} & \multirow[t]{2}{*}{ Referencia } \\
\hline & $\mathrm{Hg}^{2+}$ & $\mathrm{Cd}^{2+}$ & \\
\hline Carbones oxidados-xantados (CX) & $84^{\mathrm{a}}, 12^{\mathrm{b}}$ & $83^{\mathrm{a}}, 12^{\mathrm{b}}$ & Este trabajo \\
\hline Carbonizado de corteza de pino & & $0,34^{c}$ & \multirow{3}{*}{ Mohan et al. 2007} \\
\hline Carbonizado de madera de roble & & $0,37^{c}$ & \\
\hline Carbonizado de corteza de roble & & $5,4^{\mathrm{c}}$ & \\
\hline Carbonizado magnético de madera de roble & & $3,0^{\mathrm{d}}$ & \multirow{2}{*}{ Mohan et al. 2014} \\
\hline Carbonizado magnético de corteza de roble & & $8,3^{d}$ & \\
\hline Carbonizado de estiércol de cerdo & & $117^{\mathrm{e}}$ & \multirow{2}{*}{ Kolodynska et al. 2012} \\
\hline Carbonizado de estiércol de vaca & & $115^{\mathrm{e}}$ & \\
\hline Carbonizado digerido de azucar de remolacha & & $49^{f}$ & Inyang et al. 2012 \\
\hline CA de madera de roble & & $3,1^{\mathrm{g}}$ & Hajati et al. 2015 \\
\hline Nanocompuesto de óxido magnético de grafeno & & $91^{\mathrm{h}}$ & Visa et al. 2010 \\
\hline Carbonizado de hojas de Rosmarinus officinalis & $588^{i}$ & & Erhayem et al. 2015 \\
\hline CA de desechos de fertilizantes & & $3,6^{\mathrm{j}}$ & Mohan et al. 2001* \\
\hline CA de residuos de sagú & $55,6^{\mathrm{k}}$ & & Kadirvelu et al. 2004* \\
\hline CA de cáscara de nuez impregnada con $\mathrm{ZnCl}_{2}$ 1:0.5 p/p & $151^{l}$ & & Zabihi et al. 2010* \\
\hline CA de turba de coco & $154^{\mathrm{m}}$ & & Namasivayam \& Kadirvelu, 1999* \\
\hline CA de Terminalia catappa & $94^{\mathrm{n}}$ & & Inbaraj \& Sulochana, 2006* \\
\hline CA de residuos de la producción de antibióticos & $129^{\circ}$ & & Budinova et al. 2008* \\
\hline CA de carbón Mengen (Turquía) & $92^{p}$ & & \multirow{6}{*}{ Ekinci et al. 2002* } \\
\hline CA de carbón Seyitomer (Turquía) & $56^{p}$ & & \\
\hline CA de carbón Some (Turquía) & $105^{p}$ & & \\
\hline CA de carbón Bolluca (Turquía) & $37^{P}$ & & \\
\hline CA de cuesco de semilla de durazno & $153^{p}$ & & \\
\hline CA de furfural & $174^{\mathrm{P}}$ & & \\
\hline $\begin{array}{l}\text { CA de biomasa leñosa de abedul impregnada con } \mathrm{H}_{3} \mathrm{PO}_{4} \\
\text { pirolizada a } 700^{\circ} \mathrm{C} \text { en vapor }\end{array}$ & $160^{\mathrm{q}}$ & & Budinova et al. 2006* \\
\hline CA de cuescos de Ceiba pentandra & $26^{r}$ & & \multirow{3}{*}{ Rao et al. 2009* } \\
\hline CA de cuescos de Phaseolus aureus & $24^{r}$ & & \\
\hline CA de desechos de Cicer arietinum & $23^{r}$ & & \\
\hline Carbón activado & $60^{s}$ & & \multirow{2}{*}{ Zhu et al. 2009* } \\
\hline CA con etilendiamina & $122^{\mathrm{s}}$ & & \\
\hline Carbón activado & $578^{t}$ & & \multirow{2}{*}{ Asasian et al. 2014* } \\
\hline $\mathrm{CA}$ impregnado con $\mathrm{SO}_{2}$ & $523^{t}$ & & \\
\hline Bagacillo activado con vapor, $\mathrm{SO}_{2}$ y $\mathrm{H}_{2} \mathrm{~S}$ & $227^{\mathrm{u}}$ & & Anoop Krishnan \& Anirudhan, 2002* \\
\hline Ceniza de cáscara de arroz & $9,3^{x}$ & & Feng et al. 2004* \\
\hline Cáscara de arroz tratada con ácido sulfúrico & $385^{y}$ & & El-Shafey, 2010* \\
\hline Carbón activado comercial & $4,1^{2}$ & & \multirow{2}{*}{ Lu et al. 2014* } \\
\hline CA oxidado con $\mathrm{H}_{2} \mathrm{O}_{2} 1: 2(\mathrm{p} / \mathrm{v})$ & $5,2^{2}$ & & \\
\hline
\end{tabular}


Continuación Tabla 4.

\begin{tabular}{|l|c|c|c|}
\hline $\begin{array}{l}\text { CA de cuesco de palma impregnado con líquidos } \\
\text { iónicos }\end{array}$ & $83^{\$}$ & Ismaiel et al. 2013* \\
\hline Coque activado & $316^{\&}$ & & Li et al. 2013* \\
\hline Coque activado funcionalizado con tioles & $695^{\&}$ & & Balsamo et al. 2011 \\
\hline Cenizas de combustión de carbón & & $0,5-4,3^{\#}$ & Bal \\
\hline
\end{tabular}

*Tomado de Hadi et al. 2015. La concentración metálica de los experimentos, mg/L (a menos que se indique otra unidad), fue: ${ }^{\mathrm{a}} 998 ;{ }^{\mathrm{b}} 124 ;{ }^{\mathrm{c}} 10000 ;{ }^{\mathrm{d}} 1-100 ;{ }^{\mathrm{e}} 207-621 ;{ }^{\mathrm{f}} 5-600 ;{ }^{\mathrm{g}} 6-20 ;{ }^{\mathrm{h}} 0.0005$ a $0.01 \mathrm{~mol} / \mathrm{L} ;{ }^{\mathrm{i}} 100-400 ;{ }^{\mathrm{j}} 10^{-3}-10^{-2} \mathrm{M} ;{ }^{\mathrm{k}} 40$ y $60 ;{ }^{\mathrm{l}} 9.7-107 ;{ }^{\mathrm{m}}$ $10-40 ;{ }^{\mathrm{n}} 5-60 ;{ }^{\mathrm{o}, \mathrm{p}, \mathrm{q}} 10-40 ;{ }^{\mathrm{r}} 10-150 ;{ }^{\mathrm{s}} 20-80 ;{ }^{\mathrm{t}} 50-800 ;{ }^{\mathrm{u}} 50-1000 ;{ }^{\mathrm{x}} 1-350 ;{ }^{\mathrm{y}} 200 ;{ }^{\mathrm{z}} 0.1-18 ;{ }^{\mathrm{s}} 10-200 ;{ }^{\mathrm{k}} 0-100 ;{ }^{\#} 5-50$.

\section{BIBLIOGRAFÍA}

1. AGENCY FOR TOXIC SUBSTANCES AND DISEASE REGISTRY - ATDSR-. 2014. Carbon Disulfide. Octubre21.2014 Disponible desde Internet en: http://www. atsdr.cdc.gov $/ \mathrm{mmg} / \mathrm{mmg}$.asp?id $=470 \&$ tid $=84$ (con acceso 16/04/2015).

2. ANILLO-CORREA, R.; COLPAS-CASTILLO, F.; MEZAFUENTES, E. 2013. Aumento del contenido de ácidos húmicos en un carbón de bajo rango a través de la oxidación con aire y con peróxido de hidrogeno o ácido nítrico. Quím. Nova (Brasil). 36(3):387-392.

3. ANOOP KRISHNAN, K.; ANIRUDHAN, T.S. 2002. Removal of mercury(II) from aqueous solutions and chlor-alkali industry effluent by steam activated and sulphurised activated carbons prepared from bagasse pith: kinetics and equilibrium studies. J. Hazard. Mater. (Holanda). 92(2):161-183.

4. ASASIAN, N.; KAGHAZCHI, T.; FARAMARZI, A.; HAKIMI-SIBONI, A.; ASADI-KESHEH, R.; KAVAND, M.; MOHTASHAMI, S.A. 2014. Enhanced mercury adsorption capacity by sulfurization of activated carbon with $\mathrm{SO}_{2}$ in a bubbling fluidized bed reactor. J. Taiwan Inst. Chem. Eng. (Holanda). 45(4):1588-1596.

5. BAILEY, S.E.; OLIN, T.J.; BRICKA, R.M.; ADRIAN, D.D. 1999. A review of potentially low-cost sorbents for heavy metals. Wat. Res. (Holanda). 33:2469-2479.

6. BALSAMO, M.; DI NATALE, F; ERTO, A.; LANCIA, A.; MONTAGNARO, F.; SANTORO, L. 2011. Cadmium adsorption by coal combustion ashes-based sorbents-Relationship between sorbent properties and adsorption capacity. J. Hazard. Mater. 187(1-3):371378.

7. BUDINOVA, T.; EKINCI, E.; YARDIM, F.; GRIMM, A.; BJORNBOM, E.; MINKOVA, V.; GORANOVA, M.
2006. Characterization and application of activated carbon produced by $\mathrm{H}_{3} \mathrm{PO}_{4}$ and water vapor activation. Fuel Process. Technol. (Holanda). 87(10):899905.

8. BUDINOVA, T.; PETROV, N.; PARRA, J.; BALOUTZOV, V. 2008. Use of an activated carbon from antibiotic waste for the removal of $\mathrm{Hg}$ (II) from aqueous solution. J. Environ. Manage. (Holanda). 88(1):165-172.

9. CASTELLAR, G.; ANGULO, E.; ZAMBRANO, A.; CHARRIS, D. 2013. Adsorption equilibrium of methylene blue dye on activated carbon. Rev. U.D.C.A Act. \& Div. Cient. (Colombia). 16(1):263-271.

10. COLPAS-CASTILLO, F.; MEZA-FUENTES, E.; FERNÁNDEZ-MAESTRE, R.; PRIMERA-PEDROZO, O.M. 2011. Carbones activados a partir de bagazo de caña de azúcar y zuro de maíz para la adsorción de cadmio y plomo. Rev. Academ. Colomb. Cienc. Exact. Físic. Nat. 35(136):303-312.

11. EKINCI, E.; BUDINOVA, T.; YARDIM, F.; PETROV, N.; RAZVIGOROVA, M.; MINKOVA, V. 2002. Removal of mercury ion from aqueous solution by activated carbons obtained from biomass and coals. Fuel Process. Technol. 77-78:437-443.

12. EL-SHAFEY, E.I. 2010. Removal of Zn(II) and $\mathrm{Hg}$ (II) from aqueous solution on a carbonaceous sorbent chemically prepared from rice husk. J. Hazard. Mater. 175(1-3):319-327.

13. ERHAYEM, M.; AL-TOHAMI, F.; MOHAMED, R.; AHMIDA, K. 2015. Isotherm, kinetic and thermodynamic studies for the sorption of mercury (II) onto activated carbon from Rosmarinus officinalis leaves. Am. J. Anal. Chem. 6:1-10.

14. FEHRMANN, C.; POHL, P. 1993. Cadmium adsorption by the non-living biomass of microalgae grown in 
axenic mass culture. J. Appl. Phycol. (Holanda). 5(6):555-562.

15. FENG, Q.; LIN, Q.; GONG, F.; SUGITA, S.; SHOYA, M. 2004. Adsorption of lead and mercury by rice husk ash. J. Coll. Interface Sci. (Holanda). 278(1):1-8.

16. HADI, P.; TO, M.H.; HUI, C.W.; LIN, C.S.K.; MCKAY, G. 2015. Aqueous mercury adsorption by activated carbons. Wat. Res. En prensa. doi:10.1016/j.watres.2015.01.018

17. HAENEL, M. 1992. Recent progress in coal structure research. Fuel (Reino Unido). 71(11):1211-1223.

18. HAJATI, S.; GHAEDI, M.; YAGHOUBI, S. 2015. Local, cheep and nontoxic activated carbon as efficient adsorbent for the simultaneous removal of cadmium ions and malachite green: Optimization by surface response methodology. J. Ind. Engin. Chem. (Holanda). 21:760-767.

19. HAYASHI, J.I.; LI, C.Z. 2004. Structure and properties of Victorian brown coal. En: Li, C.Z. Ed. Advances in the Science of Victorian Brown Coal. Ed. Elsevier Oxford (Reino Unido). p.11-84.

20. INBARAJ, B.S.; SULOCHANA, N. 2006. Mercury adsorption on a carbon sorbent derived from fruit shell of Terminalia catappa. J. Hazard. Mater. 133(1-3):283290.

21. INYANG, M.; GAO, B.; DING, W.; YAO, Y.; XUE, Y.; ZIMMERMAN, A.R.; PULLAMMANAPPALLIL, P.; CAO, X. 2012. Removal of heavy metals from aqueous solution by biochars derived from anaerobically digested biomass. Biores. Technol. (Holanda). 110:50-56.

22. ISMAIEL, A.A.; AROUA, M.K.; YUSOFF, R. 2013. Palm shell activated carbon impregnated with task-specific ionic-liquids as a novel adsorbent for the removal of mercury from contaminated water. Chem. Eng. J. 225:306-314.

23. JAIN, R.; URBAN, L.; BALBACH, H.; WEBB, M.D. 2012. Contemporary issues in environmental assessment. En: Jain, R.; Urban, L.; Balbach, H.; Webb, M.D. (Eds.) Handbook of Env. Eng. Assess. Ed. Elsevier Oxford (Reino Unido). p.361-447.

24. KADIRVELU, K.; KAVIPRIYA, M.; KARTHIKA, C.; VENNILAMANI, N.; PATTABHI, S. 2004. Mercury (II) adsorption by activated carbon made from sago waste. Carbon N.Y. (Estados Unidos). 42(4):745-752.
25. KIM, H.; LEE, K. 1998. Application of cellulose xanthate for the removal of nickel ion from aqueous solution. J. Korean Soc. Eng. 20:247-254.

26. KOLODYNSKA, D.; WNETRZAK, R.; LEAHY, J.J.; HAYES, M.H.B.; KWAPINSKI, W.; HUBICKIA, Z. 2012. Kinetic and adsorptive characterization of biochar in metal ions removal. Chem. Engin. J. 197:295-305.

27. LAO-LUQUE, C.; SOLÉ, M.; GAMISANS, X.; VALDERRAMA, C.; DORADO, A.D. 2014. Characterization of chromium (III) removal from aqueous solutions by an immature coal (leonardite). Toward a better understanding of the phenomena involved. Clean Technol. Environ. Policy. (Reino Unido). 16(1):127-136.

28. LENNTECH. 2014. Dowex-Marathon-C. Disponible desde Internet en: http://www.lenntech.com/data-sheets/ dowex-marathon-c.pdf (con acceso 04/06/2014.

29. LI, Z.; WU, L.; LIU, H.; LAN, H.; QU, J. 2013. Improvement of aqueous mercury adsorption on activated coke by thiol-functionalization. Chem. Eng. J. 228:925-934.

30. LI, Y.; HELMREICH, B. 2014. Simultaneous removal of organic and inorganic pollutants from synthetic road runoff using a combination of activated carbon and activated lignite. Sep. Purif. Technol. (Holanda). 122:6-11.

31. LIANG, S.; GUO, X.; FENG, N.; TIAN, Q. 2009. Application of orange peel xanthate for the adsorption of $\mathrm{Pb}^{2+}$ from aqueous solutions, J. Hazard. Mater. 170(1): 425-429.

32. LU, X.; JIANG, J.; SUN, K.; WANG, J.; ZHANG, Y. 2014. Influence of the pore structure and surface chemical properties of activated carbon on the adsorption of mercury from aqueous solutions. Mar. Pollut. Bull. (Reino Unido). 78(1-2):69-76.

33. MARAÑÓN, E.; SASTRE, H. 1992. Heavy metal removal in packed beds using apple wastes. Biores. Technol. 38(1):39-43.

34. MATSUI, Y.; SAKAMOTO, A.; NAKAO, S.; TANIGUCHI, T.; MATSUSHITA, T.; SHIRASAKI, N.; SAKAMOTO, N.; YURIMOTO, H. 2014. Isotope microscopy visualization of the adsorption profile of 2-methylisoborneol and geosmin in powdered activated carbon. Environ. Sci. Technol. (Holanda). 48(18):10897-10903.

35. MOHAN, D.; GUPTA, V.K.; SRIVASTAVA, S.K.; CHANDER, S. 2001. Kinetics of mercury adsorption from 
wastewater using activated carbon derived from fertilizer waste. Coll. Surf. A Physicochem. Eng. Asp. (Reino Unido). 177(2-3):169-181.

36. MOHAN, D.; PITTMAN JR., C.U.; BRICKA, M.; SMITH, F.; YANCEY, B.; MOHAMMAD, J.; STEELE, P.H.; ALEXANDRE-FRANCO, M.F.; GÓMEZ-SERRANO, V.; GONG, H. 2007. Sorption of arsenic, cadmium, and lead by chars produced from fast pyrolysis of wood and bark during bio-oil production. J. Coll. Interf. Sci. 310(1):57-73.

37. MOHAN, D.; KUMAR, H.; SARSWAT, A.; ALEXANDREFRANCO, M.; PITTMAN JR., C.U. 2014. Cadmium and lead remediation using magnetic oak wood and oak bark fast pyrolysis bio-chars. Chem. Engin. J. 236:513-528.

38. NAMASIVAYAM, C.; KADIRVELU, K. 1999. Uptake of mercury (II) from wastewater by activated carbon from an unwanted agricultural solid by-product: coirpith. Carbon N.Y. 37(1):79-84.

39. PINTO, F.; GULYURTLU, I.; LOBO, L.S.; CABRITA, I. 1999. Effect of coal pre-treatment with swelling solvents on coal liquefaction. Fuel (Holanda). 78(6): 629-634.

40. RAO, M.M.; REDDY, D.H.K.K.; VENKATESWARLU, P.; SESHAIAH, K. 2009. Removal of mercury from aqueous solutions using activated carbon prepared from agricultural by-product/waste. J. Environ. Manage. 90(1):634-643.

41. RAO, R.A.K.; KHAN, M.A.; REHMAN, F. 2011. Batch and column studies for the removal of lead(II) Ions from aqueous solution onto lignite. Adsorp. Sci. Technol. (Reino Unido). 29(1):83-98.

42. SÁNCHEZ SOTO, P.J. 2009. Efecto del tratamiento mecánico por molienda en las propiedades texturales de pirofilita. Bol. Soc. Esp. Ceram. V. 48(2):5969.

Cómo citar:

Fernández-Maestre, R.; Colpas-Castillo, F. 2015. Adsorción de mercurio y cadmio en carbón subbituminoso oxidado-xantado, sulfonado o activado y resina sintética comercial. Rev. U.D.C.A Act. \& Div. Cient. 18(1): 241-250.
43. SHI, K.Y.; TAO, X.X.; HONG, F.F.; HE, H.; JI, Y.H.; LI, J.L. 2012. Mechanism of oxidation of low rank coal by nitric acid. J. Coal Sci. Engin. (China). 18(4):396-399.

44. SUN, Y.Z.; FU, K.M.; ZHU, H.; ZHU, T.L. 2009. Silicaalumina molar ratio and some factors effect on the synthesis of zeolites from fly ash. J. Coal Sci. Engin. 15(4):430-433.

45. TORRES-BLANCAS, T.; ROA-MORALES, G.; FALL, C.; BARRERA-DÍAZ, C.; UREÑA-NUÑEZ, F.; PAVÓNSILVA, T.B. 2013. Improving lead sorption through chemical modification of de-oiled allspice husk by xanthate. Fuel. 110:4-11.

46. VISA, M.; BOGATU, C.; DUTA. A. 2010. Simultaneous adsorption of dyes and heavy metals from multicomponent solutions using fly ash. Appl. Surf. Sci. (Reino Unido). 256(17):5486-5491.

47. WING, R.E.; DOANE, W.M.; RUSSEL, C.R. 1975. Insoluble strach xanthates: use in heavy metal removal. J. Appl. Polym. Sci. (Holanda). 19:847-854.

48. WORLD COAL ASSOCIATION. 2014. Disponible desde Internet en http://www.worldcoal.org/coal/coal-mining/ (con acceso 04/06/2014).

49. ZABIHI, M.; HAGHIGHI ASL, A.; AHMADPOUR, A. 2010. Studies on adsorption of mercury from aqueous solution on activated carbons prepared from walnut shell. J. Hazard. Mater. 174(1-3):251-256.

50. ZHU, J.; DENG, B.; YANG, J.; GANG, D. 2009. Modifying activated carbon with hybrid ligands for enhancing aqueous mercury removal. Carbon N.Y. 47(8):20142025.

Recibido: Diciembre 21 de 2014

Aceptado: Abril 25 de 2015 